\title{
MS32-01 | Chalcogen Bonding in Crystal Engineering
}

Resnati, Giuseppe (Politecnico di Milano, Milano, ITA); Scilabra, Patrick (Politecnico di Milano, Milano, ITA); Terraneo, Giancarlo (Politecnico di Milano, Milano, ITA)

The distribution of the electron density in bonded atoms of Groups 14-18 of the Periodic Table is anisotropic and regions of depleted electron density, named $\sigma$-holes, are present opposite to the covalent bonds formed by these elements [1]. The electrostatic potential at the atomic surface of these regions is frequently positive and attractive interactions with donors of electron density are formed. Group 16 elements typically form two covalent bonds and can thus have two positive $\sigma$-holes interacting with nucleophiles. According to a recent IUPAC Recommendation, these interactions are named chalcogen bond ( $\mathrm{ChB}$ ), in analogy with the halogen bond (HaB), pnictogen bond $(\mathrm{PnB})$, and the tetrel bond (TtB), the analogous interactions between nucleophiles and Group 17, 15, and 14 elements. It will be described how the ChB can be strong enough to function as an effective and robust tool in crystal engineering [2]. Sulfur, selenium, and tellurium can all form ChBs in crystalline solid, the tendency to give rise to close contacts with nucleophiles increasing with the polarizability of the chalcogen. A particular attention will be given to chalcogen containing azoles and their derivatives due to the relevance of these moieties in biosystems and molecular materials.

[1] Cavallo G., Metrangolo P., Pilati T., Resnati G., Terraneo G. (2014) Cryst. Growth Des. 14, 2697-2702.

[2] Scilabra P., Terraneo G., Resnati G., (2018) Acc. Chem. Res. In press. 